\title{
An approximation of solutions to heat equations defined by gener- alized measure theoretic Laplacians
}

\author{
TIM EHNES(D AND BEN HAMBLY
}

Abstract. We consider the heat equation defined by a generalized measure theoretic Laplacian on $[0,1]$. This equation describes heat diffusion in a bar such that the mass distribution of the bar is given by a non-atomic Borel probabiliy measure $\mu$, where we do not assume the existence of a strictly positive mass density. We show that weak measure convergence implies convergence of the corresponding generalized Laplacians in the strong resolvent sense. We prove that strong semigroup convergence with respect to the uniform norm follows, which implies uniform convergence of solutions to the corresponding heat equations. This provides, for example, an interpretation for the mathematical model of heat diffusion on a bar with gaps in that the solution to the corresponding heat equation behaves approximately like the heat flow on a bar with sufficiently small mass on these gaps.

\section{Introduction}

Let $\mu$ be a non-atomic Borel probability measure on $[0,1]$ such that $0,1 \in \operatorname{supp}(\mu)$, $\mathcal{L}^{2}([0,1], \mu)$ be the space of measurable functions $f$ such that $\int_{a}^{b} f^{2} \mathrm{~d} \mu<\infty$ and $L^{2}([0,1], \mu)$ be the corresponding Hilbert space of equivalence classes with inner product $\langle f, g\rangle_{\mu}:=\int_{0}^{1} f g \mathrm{~d} \mu$. We define

$$
\begin{gathered}
\mathcal{D}_{\mu}^{2}:=\left\{f \in C^{1}([0,1]): \exists\left(f^{\prime}\right)^{\mu} \in L^{2}([0,1], \mu):\right. \\
\left.f^{\prime}(x)=f^{\prime}(0)+\int_{0}^{x}\left(f^{\prime}\right)^{\mu}(y) \mathrm{d} \mu(y), x \in[0,1]\right\} .
\end{gathered}
$$

The Krein-Feller operator with respect to $\mu$ is given as

$$
\Delta_{\mu}: \mathcal{D}_{\mu}^{2} \subseteq L^{2}([0,1], \mu) \rightarrow L^{2}([0,1], \mu), f \mapsto\left(f^{\prime}\right)^{\mu} .
$$

This definition involves the derivative with respect to $\mu$. If a function $f$ has a representation given by

$$
f(x)=\int_{0}^{x} \frac{\mathrm{d}}{\mathrm{d} \mu} f(x) \mathrm{d} \mu(x), x \in[0,1],
$$

then $\frac{\mathrm{d}}{\mathrm{d} \mu} f$ is called the $\mu$-derivative of $f$. Consequently, in the above definition, $\left(f^{\prime}\right)^{\mu}$ is the $\mu$-derivative of $f^{\prime}$. 
This operator has been widely studied, for example with an emphasis on addressing questions of the spectral asymptotics and further analytical properties [2,3,11-20, $22,23,33,34,36,37]$, diffusion processes [27,30,31], wave equations [5] and higherdimensional generalizations $[21,35,39]$.

In order to connect these operators with diffusion equations from a physical point of view, we follow for example [26, Section 1.2] and consider a metallic rod of constant cross-sectional area oriented in the $x$-direction occupying a region from $x=0$ to $x=1$ such that all thermal quantities are constant across a section. We can thus consider the rod as one-dimensional. We investigate the conduction of heat energy on a segment from $x=a$ to $x=b$. Let the temperature at the point $x \in[a, b]$ and time $t \in[0, \infty)$ be denoted by $u(t, x)$ and the total thermal energy in the segment at time $t$ be denoted by $e_{a, b}(t)$. It is well-known that

$$
e_{a, b}(t)=\int_{a}^{b} u(t, x) \rho(x) \mathrm{d} x,
$$

assuming that the rod possesses a mass density $\rho:[0,1] \rightarrow(0, \infty)$. However, if we denote the mass distribution of the rod by $\mu$, we can write

$$
e_{a, b}(t)=\int_{a}^{b} u(t, x) \mathrm{d} \mu(x) .
$$

Hence, we can define the total heat energy even if $\mu$ has no density. The total heat energy changes only if heat energy flows through the boundaries $x=a$ and $x=b$. We deduce for the rate of change of heat energy

$$
\frac{\mathrm{d}}{\mathrm{d} t} e_{a, b}(t)=\phi(t, a)-\phi(t, b),
$$

where $\phi(t, x)$ denotes the heat flux density, which gives the rate of thermal energy flowing through $x$ at time $t$ to the right. Assuming sufficient regularity, we can rewrite (1) as

$$
\int_{a}^{b} \frac{\partial}{\partial t} u(t, x) \mathrm{d} \mu(x)=-\int_{a}^{b} \frac{\mathrm{d}}{\mathrm{d} \mu} \phi_{t}(x) \mathrm{d} \mu(x),
$$

where $\phi_{t}(x):=\phi(t, x)$ and the $\mu$-derivative was defined earlier. With $u_{t}(x):=$ $u(x, t)$, Fourier's law of heat conduction $\phi=-\frac{\partial u}{\partial x}$ gives

$$
\int_{a}^{b} \frac{\partial}{\partial t} u(t, x) \mathrm{d} \mu(x)=\int_{a}^{b} \frac{\mathrm{d}}{\mathrm{d} \mu} \frac{\mathrm{d}}{\mathrm{d} x} u_{t}(x) \mathrm{d} \mu(x) .
$$

Since this is valid for all $a, b \in[0,1], a<b$, it follows for $t \in[0, \infty)$ and $\mu$-almost all $x \in[0,1]$

$$
\frac{\partial}{\partial t} u(t, x)=\frac{\mathrm{d}}{\mathrm{d} \mu} \frac{\mathrm{d}}{\mathrm{d} x} u_{t}(x) .
$$


Applying the definition of the Krein-Feller operator leads to the generalized heat equation

$$
\frac{\partial u}{\partial t}=\Delta_{\mu} u_{t}, t \in[0, \infty)
$$

with Dirichlet boundary conditions $u(t, 0)=u(t, 1)=0$ for all $t \geq 0$ if we assume that the temperature vanishes at the boundaries or with Neumann boundary conditions $\frac{\partial u}{\partial x}(t, 0)=\frac{\partial u}{\partial x}(t, 1)=0$ if the boundaries are assumed to be perfectly insulated. This provides a physical motivation for a mass distribution having full support even if it possesses no Lebesgue density. However, it is still not clear how to interpret the equation if the support of the mass distribution is not the whole interval, in particular for singular measures, such as measures on the Cantor set.

The problem then is to describe heat flow on a rod with massless parts. Krein-Feller operators defined by measures on the classical Cantor set or, more generally, Cantorlike sets with gaps have been extensively studied in recent years (see, e.g., [1,16-19]). In this paper, we give an interpretation of a solution to (2) in the case where $\mu$ is not of full support. We approximate the solution by a sequence of solutions to heat equations defined by $\mu_{n}$ for $n \in \mathbb{N}$ such that $\mu_{n}$ is of full support and converges weakly to $\mu$ for $n \rightarrow \infty$.

To this end, let $b \in\{N, D\}$ represent the boundary condition, where $N$ denotes Neumann and $D$ Dirichlet boundary conditions and we give our basic assumption.

Assumption 1.1. Let $\left(\mu_{n}\right)_{n \in \mathbb{N}}$ be a sequence of non-atomic Borel probability measures on $[0,1]$ such that $0,1 \in \operatorname{supp}\left(\mu_{n}\right)$ and $\mu_{n} \rightarrow \mu, n \rightarrow \infty$, where $\rightarrow$ denotes weak measure convergence.

It is well-known that $\Delta_{\mu}^{b}$ is a non-positive self-adjoint operator (see, e.g., [13]) and thus the generator of a strongly continuous semigroup $\left(T_{t}^{b}\right)_{t \geq 0}$ (see, e.g., [24, Lemma 1.3.2]).

If $u_{0} \in L^{2}([0,1], \mu)$, then the unique mild solution to the initial value problem

$$
\begin{aligned}
\frac{\partial u}{\partial t}(t) & =\Delta_{\mu}^{b} u(t), t \in[0, \infty), \\
u(0) & =u_{0}
\end{aligned}
$$

is given by $u(t)=T_{t}^{b} u_{0}$ (see e.g., [10, Proposition II.6.4]).

This motivates the investigation of strong semigroup convergence. However, for different measures, the corresponding semigroups are defined on different spaces. For the special case $\operatorname{supp}(\mu)=\operatorname{supp}\left(\mu_{n}\right)=[0,1]$ for all $n \in \mathbb{N}$, the results in [6] can be applied to obtain strong semigroup convergence on the space of continuous functions on $[0,1]$. To formulate a strong semigroup convergence result without that assumption, we restrict the semigroup $\left(T_{t}^{N}\right)_{t \geq 0}$ associated to $\Delta_{\mu}^{N}$ on $L^{2}([0,1], \mu)$ to the subspace of continuous functions, denoted by $(C[0,1])_{\mu}^{N}$, which is a Banach space with the uniform norm. The semigroup $\left(T_{t}^{D}\right)_{t \geq 0}$ is restricted to the Banach 
space of continuous functions satisfying Dirichlet boundary conditions, denoted by $(C[0,1])_{\mu}^{D}$. We show that the restricted semigroup, which we denote by $\left(\bar{T}_{t}^{b}\right)_{t \geq 0}$, is, again, a strongly continuous contraction semigroup and the infinitesimal generator is given by

$$
\bar{\Delta}_{\mu}^{b} f:=\Delta_{\mu}^{b} f, \mathcal{D}\left(\bar{\Delta}_{\mu}^{b}\right):=\left\{f \in \mathcal{D}\left(\Delta_{\mu}^{b}\right): \Delta_{\mu}^{b} f \in(C[0,1])_{\mu}^{b}\right\} .
$$

Moreover, if we assume that $\operatorname{supp}(\mu) \subseteq \operatorname{supp}\left(\mu_{n}\right)$, the space $(C[0,1])_{\mu}^{b}$ can be continuously embedded in $(C[0,1])_{\mu_{n}}^{b}$, where we denote the embedding by $\pi_{n}$. Due to the Trotter-Kato approximation theorem (see, e.g., [10, Theorem I.6.1]), the strong semigroup convergence is equivalent to strong resolvent convergence and strong resolvent convergence is what we will establish. More precisely, let $f \in(C[0,1])_{\mu}^{b}, \lambda>0$ and $n \in \mathbb{N}$. We define $\bar{R}_{\lambda}^{b}:=\left(\lambda-\bar{\Delta}_{\mu}^{b}\right)^{-1}$ and $\bar{R}_{\lambda, n}^{b}:=\left(\lambda-\bar{\Delta}_{\mu_{n}}^{b}\right)^{-1}$ and prove

$$
\left\|\pi_{n} \bar{R}_{\lambda}^{b} f-\bar{R}_{\lambda, n}^{b} \pi_{n} f\right\|_{\infty} \rightarrow 0, n \rightarrow \infty .
$$

The main tool for proving (4) is the generalization of the hyperbolic functions sinh and cosh, defined by generalizing the series

$$
\sinh (z x)=\sum_{k=0}^{\infty} z^{2 k+1} \frac{x^{2 k+1}}{(2 k+1) !}, \cosh (z x)=\sum_{k=0}^{\infty} z^{2 k} \frac{x^{2 k}}{(2 k) !} .
$$

We replace $\frac{x^{k}}{k !}$ by generalized monomials defined by a measure $\mu$. This extends the theory of measure theoretic functions, developed for trigonometric functions in [1]. Then, we show that the resolvent density of the operator $\Delta_{\mu}^{b}$ is a product of such generalized hyperbolic functions. This leads to strong resolvent convergence for our sequence by proving convergence of these generalized hyperbolic functions. As a consequence we obtain our main result.

Theorem 1.2. Let $f \in(C[0,1])_{\mu}^{b}$ and $\mu_{n}$ be a sequence of measures satisfying Assumption 1.1. Then, for all $t \geq 0$

$$
\lim _{n \rightarrow \infty}\left\|\pi_{n} \bar{T}_{t}^{b} f-\bar{T}_{t, n}^{b} \pi_{n} f\right\|_{\infty}=0,
$$

uniformly on bounded time intervals.

For $f \in(C[0,1])_{\mu}^{b}$, it follows in the same way as before, that $\left\{u(t)=\bar{T}_{t}^{b} f: t \geq 0\right\}$ is the unique mild solution to the initial value problem

$$
\begin{aligned}
\frac{\partial u}{\partial t}(t) & =\bar{\Delta}_{\mu}^{b} u(t), t \in[0, \infty), \\
u(0) & =f
\end{aligned}
$$

Further, $\left\{u_{n}(t)=\bar{T}_{t, n}^{b} f: t \geq 0\right\}$ is the unique mild solution to the initial value problem

$$
\begin{aligned}
\frac{\partial u_{n}}{\partial t}(t) & =\bar{\Delta}_{\mu_{n}}^{b} u_{n}(t), t \in[0, \infty), \\
u_{n}(0) & =\pi_{n} f .
\end{aligned}
$$


Finally, combining these results and Theorem 1.2 yields

$$
\lim _{n \rightarrow \infty}\left\|\pi_{n} u(t)-u_{n}(t)\right\|_{\infty}=0
$$

uniformly on bounded time intervals.

We obtain a meaningful interpretation for the diffusion of heat in the case of a mass distribution with gaps in that the heat in a rod with mass distribution $\mu$ diffuses approximately like the heat on a rod with mass distribution $\mu_{n}$ for sufficiently large $n$.

This paper is structured as follows. In the following section, we recall definitions related to Krein-Feller operators. In Sect. 3, we introduce the concept of generalized hyperbolic functions and the connection to resolvent operators. Section 4 is devoted to the restriction of the Krein-Feller operator semigroup to the spaces $(C[0,1])_{\mu}^{b}$ for $b \in\{N, D\}$. After these preparations, in Sect. 5 we develop the central convergence results, namely the convergence of the hyperbolic functions and the strong resolvent convergence in Sect. 5.1 and finally, the strong semigroup convergence and convergence of solutions to heat equations in Sect. 5.2. In Sect. 6, we show how to apply the results in three examples. Lastly, in Sect. 7, we discuss some open problems.

\section{Preliminaries}

First, we recall the definition and some properties of the operator $\Delta_{\mu}^{b}$, where $b \in$ $\{N, D\}$ and $\mu$ is a non-atomic Borel probability measure on $[0,1]$ such that $0,1 \in$ $\operatorname{supp}(\mu)$. If $[0,1] \backslash \operatorname{supp}(\mu) \neq \emptyset$, then $[0,1] \backslash \operatorname{supp}(\mu)$ is open in $\mathbb{R}$ and can be written as

$$
[0,1] \backslash \operatorname{supp}(\mu)=\bigcup_{i \geq 1}\left(a_{i}, b_{i}\right)
$$

with $0<a_{i}<b_{i}<1, a_{i}, b_{i} \in \operatorname{supp}(\mu)$ for $i \geq 1$. We define

$$
\begin{gathered}
\mathcal{D}^{1}:=\left\{f:[0,1] \rightarrow \mathbb{R}: \text { there exists } f^{\prime} \in L^{2}\left([0,1], \lambda^{1}\right):\right. \\
\left.f(x)=f(0)+\int_{0}^{x} f^{\prime}(y) \mathrm{d} y, x \in[0,1]\right\}
\end{gathered}
$$

and $H^{1}\left([0,1], \lambda^{1}\right)$ to be the space of all $L^{2}([0,1], \mu)$-equivalence classes possessing a $\mathcal{D}^{1}$-representative. If $\mu=\lambda^{1}$ on $[0,1]$, this definition is equivalent to the definition of the Sobolev space $W_{2}^{1}$.

We observe that $H^{1}\left([0,1], \lambda^{1}\right)$ is the domain of the non-negative symmetric bilinear form $\mathcal{E}$ on $L^{2}([0,1], \mu)$ defined by

$$
\mathcal{E}(u, v)=\int_{0}^{1} u^{\prime}(x) v^{\prime}(x) \mathrm{d} x, u, v \in \mathcal{F}:=H^{1}\left([0,1], \lambda^{1}\right) .
$$


It is known (see [14, Theorem 4.1]) that $(\mathcal{E}, \mathcal{F})$ defines a Dirichlet form on $L^{2}([0,1], \mu)$. Hence, there exists an associated non-positive, self-adjoint operator $\Delta_{\mu}^{N}$ on $L^{2}([0,1], \mu)$ with $\mathcal{F}=\mathcal{D}\left(\left(-\Delta_{\mu}^{N}\right)^{\frac{1}{2}}\right)$ such that

$$
\left\langle-\Delta_{\mu}^{N} u, v\right\rangle_{\mu}=\mathcal{E}(u, v), u \in \mathcal{D}\left(\Delta_{\mu}^{N}\right), v \in \mathcal{F}
$$

and

$\mathcal{D}\left(\Delta_{\mu}^{N}\right)=\left\{f \in L^{2}([0,1], \mu): f\right.$ has a representative $\bar{f}$ with $\bar{f} \in \mathcal{D}_{\mu}^{2}$ and $\left.\bar{f}^{\prime}(0)=\bar{f}^{\prime}(1)=0\right\}$.

The operator $\Delta_{\mu}^{N}$ is called the Neumann Krein-Feller operator with respect to $\mu$. Furthermore, let $\mathcal{F}_{0}$ be the space of all $L^{2}([0,1], \mu)$-equivalence classes having a $\mathcal{D}^{1}$-representative $f$ such that $f(0)=f(1)=0$. The bilinear form defined by

$$
\mathcal{E}(u, v)=\int_{0}^{1} u^{\prime}(x) v^{\prime}(x) \mathrm{d} x, u, v \in \mathcal{F}_{0},
$$

is a Dirichlet form, too (see [14, Theorem 4.1]). Again, there exists an associated nonpositive, self-adjoint operator $\Delta_{\mu}^{D}$ on $L^{2}([0,1], \mu)$ with $\mathcal{F}_{0}=\mathcal{D}\left(\left(-\Delta_{\mu}^{D}\right)^{\frac{1}{2}}\right)$ such that

$$
\left\langle-\Delta_{\mu}^{D} u, v\right\rangle_{\mu}=\mathcal{E}(u, v), u \in\left(\Delta_{\mu}^{D}\right), v \in \mathcal{F}_{0}
$$

and

$\mathcal{D}\left(\Delta_{\mu}^{D}\right)=\left\{f \in L^{2}([0,1], \mu): f\right.$ has a representative $\bar{f}$ with $\bar{f} \in \mathcal{D}_{\mu}^{2}$ and $\left.\bar{f}(0)=\bar{f}(1)=0\right\}$.

Then, $\Delta_{\mu}^{D}$ is called the Dirichlet Krein-Feller operator with respect to $\mu$.

By Freiberg [13, Proposition 6.3, Lemma 6.7, Corollary 6.9], there exists an orthonormal basis $\left\{\varphi_{k}^{b}: k \in \mathbb{N}\right\}$ of $L^{2}([0,1], \mu)$ consisting of eigenfunctions of $-\Delta_{\mu}^{b}$ and for the related ascending ordered eigenvalues $\left\{\lambda_{k}^{b}: k \in \mathbb{N}\right\}$ we have $0 \leq \lambda_{1}^{b} \leq \lambda_{2}^{b} \leq \cdots$, where $\lambda_{1}^{D}>0$. Since $\left\{\varphi_{k}^{b}: k \geq 1\right\}$ is an orthonormal basis of $L^{2}([0,1], \mu)$, each $f \in L^{2}([0,1], \mu)$ can be written as $f=\sum_{k \geq 1} f_{k}^{b} \varphi_{k}^{b}$, where $f_{k}^{b}:=\left\langle f, \varphi_{k}^{b}\right\rangle_{\mu}, k \geq 1$. Along with the self-adjointness, we obtain the following formula, called the spectral representation of $\Delta_{\mu}^{b}$ (see, e.g., [24, Section 1.3]):

$$
\begin{aligned}
-\Delta_{\mu}^{b} f & =\sum_{k \geq 1} \lambda_{k}^{b} f_{k}^{b} \varphi_{k}^{b}, \\
\mathcal{D}\left(\Delta_{\mu}^{b}\right) & =\left\{f \in L^{2}([0,1], \mu): \sum_{k \geq 1}\left(\lambda_{k}^{b} f_{k}^{b}\right)^{2}<\infty\right\} .
\end{aligned}
$$

The spectral representation provides a direct way to introduce the associated semigroup. Define for $f \in L^{2}([0,1], \mu)$

$$
T_{t}^{b} f:=\sum_{k \geq 1} e^{-\lambda_{k}^{b} t} f_{k}^{b} \varphi_{k}^{b}, t \geq 0 .
$$


Then, $\left(T_{t}^{b}\right)_{t \geq 0}$ is a strongly continuous semigroup on $L^{2}([0,1], \mu)$ and its infinitesimal generator is $\Delta_{\mu}^{b}$ (see, e.g., [24, Lemma 1.3.2]).

\section{Generalized hyperbolic functions and the resolvent operator}

Let $b \in\{N, D\}$ and let $\mu$ be defined as before. In this section, we develop a useful representation for the resolvent density of $\Delta_{\mu}^{b}$.

Let $\lambda>0$. We consider the initial value problem

$$
\left\{\begin{array}{l}
\Delta_{\mu} g=\lambda g \\
g(0)=1, g^{\prime}(0)=0
\end{array}\right.
$$

on $L^{2}([0,1], \mu)$. The problem (9) possesses a unique solution (see [13, Lemma 5.1]), which we denote by $g_{1, N}^{\lambda}$. Further, under the initial conditions

$$
\begin{aligned}
& g(1)=1, g^{\prime}(1)=0, \\
& g(0)=0, g^{\prime}(0)=1
\end{aligned}
$$

and

$$
g(1)=0, g^{\prime}(1)=1,
$$

respectively, the above eigenvalue problems also possess unique solutions (see[13, Remark 5.2]), and we denote them by $g_{2, N}^{\lambda}, g_{1, D}^{\lambda}$ and $g_{2, D}^{\lambda}$, respectively. The resolvent density is then given as follows.

Lemma 3.1. [13, Theorem 6.1] Let $\lambda>0$. The resolvent operator $R_{\lambda}^{b}:=\left(\lambda-\Delta_{\mu}^{b}\right)^{-1}$ is well-defined and for all $f \in L^{2}([0,1], \mu)$ we have

$$
R_{\lambda}^{b} f(x)=\int_{0}^{1} \rho_{\lambda}^{b}(x, y) f(y) \mathrm{d} \mu(y), x \in[0,1],
$$

where the resolvent densities are given by

$$
\begin{aligned}
& \rho_{\lambda}^{N}(x, y)=\rho_{\lambda}^{N}(y, x):=\frac{g_{1, N}^{\lambda}(x) g_{2, N}^{\lambda}(y)}{\left(g_{1, N}^{\lambda}\right)^{\prime}(1)}, x, y \in[0,1], x \leq y, \\
& \rho_{\lambda}^{D}(x, y)=\rho_{\lambda}^{D}(y, x):=-\frac{g_{1, D}^{\lambda}(x) g_{2, D}^{\lambda}(y)}{g_{1, D}^{\lambda}(1)}, x, y \in[0,1], x \leq y .
\end{aligned}
$$

It is well-known that if $\mu=\lambda^{1}$, the solutions to (9) and (11) are given by

$$
g_{1 . N}^{\lambda}(x)=\cosh (\sqrt{\lambda} x) \text { and } g_{1 . D}^{\lambda}(x)=\frac{1}{\sqrt{\lambda}} \sinh (\sqrt{\lambda} x), x \in[0,1],
$$

respectively. We generalize the notion of hyperbolic functions by solving (9) and (11) for an arbitrary measure $\mu$ with the given initial conditions. To this end, we introduce generalized monomials as in [1]. 
Definition 3.2. For $x \in[0,1]$ we set $p_{0}(x)=q_{0}(x)=1$ and for $k \in \mathbb{N}$

$$
\begin{aligned}
& p_{k}(x):= \begin{cases}\int_{0}^{x} p_{k-1}(t) \mathrm{d} \mu(t), & \text { if } k \text { is odd, } \\
\int_{0}^{x} p_{k-1}(t) \mathrm{d} t, & \text { if } k \text { is even, }\end{cases} \\
& q_{k}(x):= \begin{cases}\int_{0}^{x} q_{k-1}(t) \mathrm{d} t, & \text { if } k \text { is odd, } \\
\int_{0}^{x} q_{k-1}(t) \mathrm{d} \mu(t), & \text { if } k \text { is even. }\end{cases}
\end{aligned}
$$

We note that for $x \in[0,1]$ and $k \geq 0$,

$$
p_{2 k+1}(x) \leq p_{2 k}(x) \leq \frac{x^{k}}{k !}, q_{2 k+1}(x) \leq p_{2 k}(x) \leq \frac{x^{k}}{k !}
$$

(see [18, Lemma 2.3]).

Definition 3.3. We define for $x \in[0,1], z \in \mathbb{R}$

$$
\sinh _{z}(x):=\sum_{k=0}^{\infty} z^{2 k+1} q_{2 k+1}(x), \cosh _{z}(x):=\sum_{k=0}^{\infty} z^{2 k} p_{2 k}(x) .
$$

By (13) for all $z \in \mathbb{R}$

$$
\left\|\sinh _{z}\right\|_{\infty} \leq z e^{z^{2}},\left\|\cosh _{z}\right\|_{\infty} \leq e^{z^{2}}
$$

Example 3.4. If $\mu=\lambda^{1}$, we have $q_{k}(x)=\frac{x^{k}}{k !}, k \geq 0$. It follows that in this case

$$
\sinh _{z}(x)=\sum_{k=0}^{\infty} z^{2 k+1} \frac{x^{2 k+1}}{(2 k+1) !}=\sinh (z x)
$$

and analogously $\cosh _{z}(x)=\cosh (z x)$.

Proposition 3.5. Let $\lambda>0$. Then, for $x \in[0,1]$, we have

$$
\begin{array}{ll}
g_{1, N}^{\lambda}(x)=\cosh _{\sqrt{\lambda}}(x), & g_{1, D}^{\lambda}(x)=\frac{1}{\sqrt{\lambda}} \sinh _{\sqrt{\lambda}}(x), \\
g_{2, N}^{\lambda}(x)=\cosh _{\sqrt{\lambda}}(1-x), & g_{2, D}^{\lambda}(x)=-\frac{1}{\sqrt{\lambda}} \sinh _{\sqrt{\lambda}}(1-x) .
\end{array}
$$


Proof. The assertion for $g_{1, D}^{\lambda}$ was proven in [18, Lemma 2.3]. The proof for $g_{1, N}^{\lambda}$ works analogously. We verify the assertion for $g_{2, N}^{\lambda}$. Let $x \in[0,1]$. Then,

$$
\begin{aligned}
\cosh _{\sqrt{\lambda}}(1-x) & =\sum_{n=0}^{\infty} \lambda^{n} p_{2 n}(1-x) \\
& =1+\sum_{n=1}^{\infty} \lambda^{n} \int_{0}^{1-x} \int_{0}^{y} p_{2 n-2}(t) \mathrm{d} \mu(t) \mathrm{d} y \\
& =1+\sum_{n=1}^{\infty} \lambda^{n} \int_{0}^{1-x} \int_{1-y}^{1} p_{2 n-2}(1-t) \mathrm{d} \mu(t) \mathrm{d} y \\
& =1-\sum_{n=1}^{\infty} \lambda^{n} \int_{x}^{1} \int_{0}^{y} p_{2 n-2}(1-t) \mathrm{d} \mu(t) \mathrm{d} y \\
& =1-\sum_{n=0}^{\infty} \lambda^{n+1} \int_{x}^{1} \int_{0}^{y} p_{2 n}(1-t) \mathrm{d} \mu(t) \mathrm{d} y .
\end{aligned}
$$

Due to estimate (13) we can use the dominated convergence theorem and obtain

$$
\begin{aligned}
\cosh _{\sqrt{\lambda}}(1-x) & =1-\lambda \int_{x}^{1} \int_{0}^{y} \sum_{n=0}^{\infty} \lambda^{n} p_{2 n}(1-t) \mathrm{d} \mu(t) \mathrm{d} y \\
& =1-\lambda \int_{x}^{1} \int_{0}^{y} \cosh _{\sqrt{\lambda}}(1-t) \mathrm{d} \mu(t) \mathrm{d} y .
\end{aligned}
$$

We set $f(x):=\cosh _{\sqrt{\lambda}}(1-x), x \in[0,1]$ and get

$$
f(x)=1-\lambda \int_{x}^{1} \int_{0}^{y} f(t) \mathrm{d} \mu(t) \mathrm{d} y, x \in[0,1]
$$

and in particular

$$
f(0)=1-\lambda \int_{0}^{1} \int_{0}^{y} f(t) \mathrm{d} \mu(t) \mathrm{d} y .
$$

It follows that, for $x \in[0,1]$,

$$
f(x)-f(0)=\lambda \int_{0}^{x} \int_{0}^{y} f(t) \mathrm{d} \mu(t) \mathrm{d} y .
$$

The latter equation can be written as $\Delta_{\mu} f=\lambda f$. It remains to verify the initial conditions. Obviously, $f(1)=\cosh _{\sqrt{\lambda}}(0)=1$. Using (13) again, we have

$$
f^{\prime}(1)=-\sum_{n=1}^{\infty} \lambda^{n} p_{2 n-1}(0)=0 .
$$

The proof for $g_{2, D}^{\lambda}$ follows using the same ideas. 
This leads to the following representation for the resolvent density:

Corollary 3.6. Let $\lambda>0$. We have for $x, y \in[0,1], x \leq y$,

$$
\begin{aligned}
& \rho_{\lambda}^{N}(x, y)=\rho_{\lambda}^{N}(y, x)=\left(\cosh _{\sqrt{\lambda}}^{\prime}(1)\right)^{-1} \cosh _{\sqrt{\lambda}}(x) \cosh _{\sqrt{\lambda}}(1-y), \\
& \rho_{\lambda}^{D}(x, y)=\rho_{\lambda}^{D}(y, x)=\frac{1}{\sqrt{\lambda}}\left(\sinh _{\sqrt{\lambda}}(1)\right)^{-1} \sinh _{\sqrt{\lambda}}(x) \sinh _{\sqrt{\lambda}}(1-y) .
\end{aligned}
$$

\section{The restricted semigroup}

Let $b \in\{N, D\}$ and let $\mu$ be defined as before. It is well-known that $\Delta_{\mu}^{b}$ is the generator of a strongly continuous Markovian semigroup $\left(T_{t}^{b}\right)_{t \geq 0}$ of contractions on $L^{2}([0,1], \mu)$.

Definition 4.1. For $(t, x, y) \in(0, \infty) \times[0,1] \times[0,1]$, we define

$$
p_{t}^{b}(x, y):=\sum_{k=1}^{\infty} e^{-\lambda_{k}^{b} t} \varphi_{k}^{b}(x) \varphi_{k}^{b}(y)
$$

This is called the heat kernel of $\Delta_{\mu}^{b}$.

The heat kernel is the integral kernel of the semigroup $\left(T_{t}^{b}\right)_{t \geq 0}$. That is, for $t>0$ and $f \in L^{2}([0,1], \mu)$, we can write

$$
T_{t}^{b} f(x)=\int_{0}^{1} p_{t}^{b}(x, y) f(y) \mathrm{d} \mu(y), x \in[0,1] .
$$

In this section, we restrict these semigroups to appropriate spaces of equivalence classes of continuous functions.

Definition 4.2. (i) We define $(C[0,1])_{\mu}^{N}$ as the set of all $L^{2}([0,1], \mu)$-equivalence classes possessing a continuous representative, formally

$$
(C[0,1])_{\mu}^{N}:=\left\{f \in L^{2}([0,1], \mu): f \text { possesses a continuous representative }\right\} .
$$

(ii) We further define $(C[0,1])_{\mu}^{D}$ as the set of all $L^{2}([0,1], \mu)$-equivalence classes possessing a continuous representative that satisfies Dirichlet boundary conditions, formally

$(C[0,1])_{\mu}^{D}:=\left\{f \in L^{2}([0,1], \mu): f\right.$ possesses a continuous representative $\bar{f}$

$$
\text { such that } \bar{f}(0)=\bar{f}(1)=0\} \text {. }
$$

The space $(C[0,1])_{\mu}^{b}$ is a Banach space with the norm $\|f\|_{(C[0,1])_{\mu}^{b}}:=\left\|\left.f\right|_{\operatorname{supp}(\mu)}\right\|_{\infty}$. Note that

$$
\|f\|_{(C[0,1])_{\mu}^{b}}=\|\widetilde{f}\|_{\infty}
$$


where $\tilde{f}$ is the continuous representative of $f$ that is affine on all intervals in $[0,1] \backslash$ $\operatorname{supp}(\mu)$. To simplify the notation, we henceforth write $\|f\|_{\infty}$ for $\|f\|_{(C[0,1])_{\mu}^{b}}$.

Let $u=\sum_{k \geq 1} u_{k}^{b} \varphi_{k}^{b} \in L^{2}([0,1], \mu)$ and let $t>0$. From $\left(f_{k}^{b}\right)_{k \geq 1} \in l^{2}(\mathbb{N})$ it follows that $\left(\left(\lambda_{k}^{b}\right)^{n} e^{-\lambda_{k}^{b} t} f_{k}^{b}\right)_{k \geq 1} \in l^{2}(\mathbb{N})$ and thus by (7) and (8)

$$
\left(-\Delta_{\mu}^{b}\right)^{k} T_{t}^{b} u=\sum_{k \geq 1}\left(\lambda_{k}^{b}\right)^{k} e^{-\lambda_{k}^{b} t} u_{k}^{b} \varphi_{k}^{b} \in L^{2}([0,1], \mu)
$$

and especially $T_{t}^{b} u \in \mathcal{D}\left(\Delta_{\mu}^{b}\right)$. Hence, the following inclusion holds:

$$
T_{t}^{b}\left((C[0,1])_{\mu}^{b}\right) \subseteq(C[0,1])_{\mu}^{b}
$$

This motivates the definition of the restricted semigroup $\left(\bar{T}_{t}^{b}\right)_{t \geq 0}:=\left(\left(T_{t}^{b}\right)_{\left.\right|_{(C[0,1])_{\mu}^{b}}}\right)_{t \geq 0}$, which is for $t \geq 0$ defined by

$$
\bar{T}_{t}^{b}:(C[0,1])_{\mu}^{b} \rightarrow(C[0,1])_{\mu}^{b}, \bar{T}_{t}^{b} f=T_{t}^{b} f .
$$

When evaluating an element of $(C[0,1])_{\mu}^{b}$ pointwise, we always evaluate the representative that is affine on all intervals in $[0,1] \backslash \operatorname{supp}(\mu)$.

The goal of this section is to show that $\left(\bar{T}_{t}^{b}\right)_{t \geq 0}$ again defines a strongly continuous contraction semigroup. It is obvious that the semigroup property holds. Note that by the Markov property of $\left(T_{t}^{b}\right)_{t \geq 0}$ for $g \in(C[0,1])_{\mu}^{N}$

$$
\left|T_{t}^{b} g(x)\right|=\left|\int_{0}^{1} p_{t}^{b}(x, y) g(y) \mathrm{d} \mu(y)\right| \leq\|g\|_{\infty}\left|\int_{0}^{1} p_{t}^{b}(x, y) \mathrm{d} \mu(y)\right| \leq\|g\|_{\infty}, x \in[0,1] .
$$

Hence, $\left(\bar{T}_{t}^{b}\right)_{t \geq 0}$ is a semigroup of contractions. It remains to prove the strong continuity. To this end, we need some preparations. We write $\mathcal{E}(f, f):=\mathcal{E}(f)$ and $\|f\|_{\mu}^{2}:=\int_{0}^{1} f(x)^{2} \mathrm{~d} \mu(x)$.

Lemma 4.3. If $f \in \mathcal{F}$, then

$$
\|f\|_{\infty} \leq \mathcal{E}(f)^{\frac{1}{2}}+\|f\|_{\mu} .
$$

Proof. Let $f \in \mathcal{F}$. Then, by the Cauchy-Schwarz inequality for all $x, y \in[0,1]$

$$
|f(x)-f(y)|=\left|\int_{x}^{y} f^{\prime}(z) \mathrm{d} z\right| \leq\left(\int_{x}^{y}\left(f^{\prime}\right)^{2}(z) \mathrm{d} z\right)^{\frac{1}{2}}|x-y|^{\frac{1}{2}}=\mathcal{E}(f)^{\frac{1}{2}}|x-y|^{\frac{1}{2}} .
$$

It follows by the reversed triangle inequality and by $|x-y| \leq 1$

$$
|f(x)| \leq|f(y)|+\mathcal{E}(f)^{\frac{1}{2}} .
$$


Further, by integrating of $y$ w.r.t. $\mu$,

$$
|f(x)| \leq \int_{0}^{1}|f(y)| \mathrm{d} \mu(y)+\mathcal{E}(f)^{\frac{1}{2}}
$$

and finally by the Cauchy-Schwarz inequality

$$
|f(x)| \leq\|f\|_{\mu}+\mathcal{E}(f)^{\frac{1}{2}} .
$$

Lemma 4.4. Let $f \in(C[0,1])_{\mu}^{b}$. Then, $\lim _{t \rightarrow 0}\left\|T_{t}^{b} f-f\right\|_{\infty}=0$.

Proof. We follow the proof of [28, Proposition 5.2.6]. Let $f \in \mathcal{F}$. By Lemma 4.3 and [28, Lemma B.2.4],

$$
\begin{aligned}
\lim _{t \rightarrow 0}\left\|T_{t}^{b} f-f\right\|_{\infty} & \leq \lim _{t \rightarrow 0} \mathcal{E}\left(T_{t}^{b} f-f\right)^{\frac{1}{2}}+\left\|T_{t}^{b} f-f\right\|_{\mu} \\
& \leq \lim _{t \rightarrow 0} 2^{\frac{1}{2}}\left(\mathcal{E}\left(T_{t}^{b} f-f\right)+\left\|T_{t}^{b} f-f\right\|_{\mu}^{2}\right)^{\frac{1}{2}} \\
& =0 .
\end{aligned}
$$

By the fact that $\mathcal{F}$ is dense in $(C[0,1])_{\mu}^{N}$ and that, for $t \geq 0, T_{t}^{N}$ is continuous on $(C[0,1])_{\mu}^{N}$, we obtain the assertion for $b=N$. To verify the case $b=D$, we prove that $\mathcal{F}_{0}$ is dense in $(C[0,1])_{\mu}^{D}$. Let $f \in(C[0,1])_{\mu}^{D}$. Then, by the density of $\mathcal{F}$ in $(C[0,1])_{\mu}^{N}$, there exists a sequence $\left(f_{n}\right)_{n \in \mathbb{N}}$ with $f_{n} \in \mathcal{F}$ for each $n \in \mathbb{N}$ such that

$$
\left\|f-f_{n}\right\|_{\infty} \rightarrow 0, n \rightarrow \infty
$$

We define for $n \in \mathbb{N}$

$$
f_{n, 0}(x):=f_{n}(x)-f_{n}(0)-x\left(f_{n}(1)-f_{n}(0)\right), x \in[0,1],
$$

which is an element of $\mathcal{F}_{0}$. Further, we have that

$$
f_{0}(x):=f(x)-f(0)-x(f(1)-f(0))=f(x), x \in[0,1],
$$

since $f$ satisfies Dirichlet boundary conditions. This along with (16) implies for $n \in \mathbb{N}$

$$
\begin{aligned}
\lim _{n \rightarrow \infty} & \left\|f_{n, 0}-f\right\|_{\infty} \\
= & \lim _{n \rightarrow \infty}\left\|f_{n, 0}-f_{0}\right\|_{\infty} \\
\leq & \lim _{n \rightarrow \infty} \sup _{x \in[0,1]}\left|f_{n}(x)-f(x)\right|+\left|f_{n}(0)-f(0)\right| \\
& +\left|x\left(f_{n}(1)-f_{n}(0)-(f(1)-f(0))\right)\right| \\
= & 0 .
\end{aligned}
$$

The main result of this section now follows immediately.

Corollary 4.5. $\left(\bar{T}_{t}^{b}\right)_{t \geq 0}$ is a strongly continuous contraction semigroup on $(C[0,1])_{\mu}^{b}$. 


\section{Convergence results}

\subsection{Strong resolvent convergence}

Let $\mu$ be defined as before and let $F$ be the distribution function of $\mu$. Further, let $\left(\mu_{n}\right)_{n \in \mathbb{N}}$ satisfy Assumption 1.1 and let $F_{n}$ be the distribution function of $\mu_{n}$ for $n \in \mathbb{N}$.

First, we give convergence results for the generalized hyperbolic functions introduced in Sect. 3 using results from [19]. Let $p_{k}, q_{k}, k \in \mathbb{N}$ be defined by $\mu$ and $p_{k, n}, q_{k, n}, k \in \mathbb{N}$ be defined by $\mu_{n}$ for $n \in \mathbb{N}$.

Lemma 5.1. [19, Lemma 3.1] For $x \in[0,1]$ and $k, n \in \mathbb{N}$ we have

$$
\begin{aligned}
\left|q_{2 k}(x)-q_{2 k, n}(x)\right| & \leq 2 \frac{\left\|F-F_{n}\right\|_{\infty} x^{k}}{(k-1) !}, \\
\left|p_{2 k}(x)-p_{2 k, n}(x)\right| & \leq 2 \frac{\left\|F-F_{n}\right\|_{\infty} x^{k}}{(k-1) !}, \\
\left|q_{2 k+1}(x)-q_{2 k+1, n}(x)\right| & \leq 2 \frac{\left\|F-F_{n}\right\|_{\infty} x^{k}}{(k-1) !}, \\
\left|p_{2 k+1}(x)-p_{2 k+1, n}(x)\right| & \leq 2 \frac{\left\|F-F_{n}\right\|_{\infty} x^{k}}{(k-1) !} .
\end{aligned}
$$

Remark 5.2. Since the distribution function of $\mu$ is continuous, weak measure convergence implies uniform convergence of the corresponding distribution functions (see [4, Section 8.1]), which is the condition in [19, Lemma 3.1].

For $z \in \mathbb{R}$ let $\cosh _{z}$, $\sinh _{z}$ be defined by $\mu$ and $\cosh _{z, n}$, $\sinh _{z, n}$ be defined by $\mu_{n}$ for $n \in \mathbb{N}$. We obtain a result for the generalized hyperbolic functions, comparable to that for the trigonometric functions in [19].

Lemma 5.3. Let $z \in \mathbb{R}$. Then,

$$
\begin{aligned}
&\left\|\cosh _{z}-\cosh _{z, n}\right\|_{\infty} \leq 2 z^{2} e^{z^{2}}\left\|F-F_{n}\right\|_{\infty}, \\
&\left\|\cosh _{z}^{\prime}-\cosh _{z, n}^{\prime}\right\|_{\infty} \leq\left(z^{2}+2 z^{4} e^{z^{2}}\right)\left\|F-F_{n}\right\|_{\infty}, \\
&\left\|\sinh _{z}-\sinh _{z, n}\right\|_{\infty} \leq 2 z^{3} e^{z^{2}}\left\|F-F_{n}\right\|_{\infty} .
\end{aligned}
$$


Proof. Let $x \in[0,1]$ and $n \in \mathbb{N}$. Then,

$$
\begin{aligned}
\left|\cosh _{z}(x)-\cosh _{z, n}(x)\right| & \leq \sum_{k=1}^{\infty}\left|p_{2 k}(x)-p_{2 k, n}(x)\right| z^{2 k} \\
& \leq \sum_{k=1}^{\infty} \frac{2\left\|F-F_{n}\right\|_{\infty}}{(k-1) !} z^{2 k} \\
& =\sum_{k=0}^{\infty} \frac{2\left\|F-F_{n}\right\|_{\infty}}{k !} z^{2 k+2} \\
& =2 z^{2} e^{z^{2}}\left\|F-F_{n}\right\|_{\infty} .
\end{aligned}
$$

Further, note that

$$
\cosh _{z}^{\prime}(x)=\sum_{k=1}^{\infty} p_{2 k-1}(x) z^{2 k}
$$

and

$$
\left|p_{1}(x)-p_{1, n}(x)\right|=\left|\mu([0, x])-\mu_{n}([0, x])\right| \leq\left\|F-F_{n}\right\|_{\infty} .
$$

With that,

$$
\begin{aligned}
\left|\cosh _{z}^{\prime}(x)-\cosh _{z, n}^{\prime}(x)\right| & \leq \sum_{k=1}^{\infty}\left|p_{2 k-1}(x)-p_{2 k-1, n}(x)\right| z^{2 k} \\
& \leq\left(z^{2}+2 \sum_{k=2}^{\infty} \frac{z^{2 k}}{(k-2) !}\right)\left\|F-F_{n}\right\|_{\infty} \\
& \leq\left(z^{2}+2 z^{4} e^{z^{2}}\right)\left\|F-F_{n}\right\|_{\infty} .
\end{aligned}
$$

Finally,

$$
\begin{aligned}
\left|\sinh _{z}(x)-\sinh _{z, n}(x)\right| & \leq \sum_{k=1}^{\infty}\left|q_{2 k+1}(x)-q_{2 k+1, n}(x)\right| z^{2 k+1} \\
& \leq \sum_{k=1}^{\infty} \frac{2\left\|F-F_{n}\right\|_{\infty}}{(k-1) !} z^{2 k+1} \\
& \leq \sum_{k=0}^{\infty} \frac{2\left\|F-F_{n}\right\|_{\infty}}{k !} z^{2 k+3} \\
& \leq 2 z^{3} e^{z^{2}}\left\|F-F_{n}\right\|_{\infty} .
\end{aligned}
$$

We turn to the main result of this section. For $b \in\{N, D\}$ and $\lambda>0$, let $R_{\lambda}^{b}$ be defined by $\mu$ and $R_{\lambda, n}^{b}$ be defined by $\mu_{n}$. We assume $\operatorname{supp}(\mu) \subseteq \operatorname{supp}\left(\mu_{n}\right)$ for all $n \in \mathbb{N}$. Then, the mapping

$$
\pi_{n}:(C[0,1])_{\mu}^{b} \rightarrow(C[0,1])_{\mu_{n}}^{b}, f \mapsto f
$$


defines an embedding, where $f \in(C[0,1])_{\mu_{n}}^{b}$ denotes the $L^{2}\left([0,1], \mu_{n}\right)$-equivalence class of the representative of $f \in(C[0,1])_{\mu}^{b}$ that is affine on each interval $I \subseteq$ $\operatorname{supp}\left(\mu_{n}\right) \backslash \operatorname{supp}(\mu)$.

Theorem 5.4. Let $\lambda>0$. Then, for all $f \in(C[0,1])_{\mu}^{b}$,

$$
\lim _{n \rightarrow \infty}\left\|R_{\lambda, n}^{b} \pi_{n} f-\pi_{n} R_{\lambda}^{b} f\right\|_{\infty}=0
$$

Proof. We simplify the notation in this proof by omitting all embeddings. If we evaluate on $\operatorname{supp}\left(\mu_{n}\right) \backslash \operatorname{supp}(\mu)$, we always evaluate the representative that is affine on each interval $I \subseteq \operatorname{supp}\left(\mu_{n}\right) \backslash \operatorname{supp}(\mu)$. First, we consider the case $b=N$. Let $\lambda>0$, $n \in \mathbb{N}, x, y \in[0,1]$ with $x \leq y$. Using the triangle inequality,

$$
\begin{aligned}
& \left|\rho_{\lambda}^{N}(x, y)-\rho_{\lambda, n}^{N}(x, y)\right| \\
& \leq\left|\left(\cosh _{\sqrt{\lambda}}^{\prime}(1)\right)^{-1}-\left(\cosh _{\sqrt{\lambda}, n}^{\prime}(1)\right)^{-1}\right|\left|\cosh _{\sqrt{\lambda}}(x) \cosh _{\sqrt{\lambda}}(1-y)\right| \\
& +\left|\cosh _{\sqrt{\lambda}}(x)-\cosh _{\sqrt{\lambda, n}}(x)\right|\left|\left(\cosh _{\sqrt{\lambda}, n}^{\prime}(1)\right)^{-1} \cosh _{\sqrt{\lambda}}(1-y)\right| \\
& +\left|\cosh _{\sqrt{\lambda}}(1-y)-\cosh _{\sqrt{\lambda}, n}(1-y)\right|\left|\left(\cosh _{\sqrt{\lambda}, n}^{\prime}(1)\right)^{-1} \cosh _{\sqrt{\lambda}, n}(x)\right| .
\end{aligned}
$$

We have

$$
\cosh _{\sqrt{\lambda}}^{\prime}(1)=\sum_{n=1}^{\infty} \lambda^{n} p_{2 n-1}(1) \geq \lambda p_{1}(1)=\lambda
$$

and similarly $\cosh _{\sqrt{\lambda, n}}^{\prime}(1) \geq \lambda$. Applying this along with Lemma 5.3, we get

$$
\begin{aligned}
\left|\left(\cosh _{\sqrt{\lambda}}^{\prime}(1)\right)^{-1}-\left(\cosh _{\sqrt{\lambda, n}}^{\prime}(1)\right)^{-1}\right| & =\left|\frac{\cosh _{\sqrt{\lambda}, n}^{\prime}(1)-\cosh _{\sqrt{\lambda}}^{\prime}(1)}{\cosh _{\sqrt{\lambda}}^{\prime}(1) \cosh _{\sqrt{\lambda}, n}^{\prime}(1)}\right| \\
& \leq \frac{\left(\lambda+2 \lambda^{2} e^{\lambda}\right)\left\|F-F_{n}\right\|_{\infty}}{\lambda^{2}}
\end{aligned}
$$

and thus with (14)

$$
\begin{aligned}
& \left|\left(\cosh _{\sqrt{\lambda}}^{\prime}(1)\right)^{-1}-\left(\cosh _{\sqrt{\lambda}, n}^{\prime}(1)\right)^{-1}\right|\left|\cosh _{\sqrt{\lambda}}(x) \cosh _{\sqrt{\lambda}}(1-y)\right| \\
& \quad \leq \frac{\left(e^{2 \lambda}+2 \lambda e^{3 \lambda}\right)\left\|F-F_{n}\right\|_{\infty}}{\lambda} .
\end{aligned}
$$

For the second term on the right-hand side of inequality (18), we calculate

$$
\left|\cosh _{\sqrt{\lambda}}(x)-\cosh _{\sqrt{\lambda}, n}(x)\right|\left|\left(\cosh _{\sqrt{\lambda}, n}^{\prime}(1)\right)^{-1} \cosh _{\sqrt{\lambda}}(1-y)\right| \leq 2 e^{2 \lambda}\left\|F-F_{n}\right\|_{\infty} .
$$


Treating the third term analogously and using the above calculations in (18) yields

$$
\begin{aligned}
& \lim _{n \rightarrow \infty} \max _{x \in[0,1]}\left|\rho_{\lambda}^{N}(x, y)-\rho_{\lambda, n}^{N}(x, y)\right| \\
& \quad \leq \lim _{n \rightarrow \infty} \frac{\left(e^{2 \lambda}+2 \lambda e^{3 \lambda}\right)\left\|F-F_{n}\right\|_{\infty}}{\lambda}+4 e^{2 \lambda}\left\|F-F_{n}\right\|_{\infty} \\
& \quad=\lim _{n \rightarrow \infty}\left(\frac{1}{\lambda}+2 e^{\lambda}+4\right) e^{2 \lambda}\left\|F-F_{n}\right\|_{\infty} \\
& \quad=0 .
\end{aligned}
$$

Further, by (14) and (19),

$$
\begin{aligned}
& \left|\int_{0}^{1} \rho_{\lambda}^{N}(x, y) f(y) \mathrm{d} \mu(y)-\int_{0}^{1} \rho_{\lambda}^{N}(x, y) f(y) \mathrm{d} \mu_{n}(y)\right| \\
& \quad \leq\left|\left(\cosh _{\sqrt{\lambda}}^{\prime}(1)\right)^{-1} \cosh _{\sqrt{\lambda}}(x)\right| \\
& \quad \cdot\left|\int_{0}^{1} \cosh _{\sqrt{\lambda}}(1-y) f(y) \mathrm{d} \mu(y)-\int_{0}^{1} \cosh _{\sqrt{\lambda}}(1-y) f(y) \mathrm{d} \mu_{n}(y)\right| \\
& \leq \frac{e^{\lambda}}{\lambda}\left|\int_{0}^{1} \cosh _{\sqrt{\lambda}}(1-y) f(y) \mathrm{d} \mu(y)-\int_{0}^{1} \cosh _{\sqrt{\lambda}}(1-y) f(y) \mathrm{d} \mu_{n}(y)\right| .
\end{aligned}
$$

Due to weak measure convergence,

$$
\lim _{n \rightarrow \infty} \int_{0}^{1} \cosh _{\sqrt{\lambda}}(1-y) f(y) \mathrm{d} \mu_{n}(y)-\int_{0}^{1} \cosh _{\sqrt{\lambda}}(1-y) f(y) \mathrm{d} \mu(y)=0
$$

and consequently,

$$
\lim _{n \rightarrow \infty} \max _{x \in[0,1]}\left|\int_{0}^{1} \rho_{\lambda}^{N}(x, y) f(y) \mathrm{d} \mu(y)-\int_{0}^{1} \rho_{\lambda}^{N}(x, y) f(y) \mathrm{d} \mu_{n}(y)\right|=0 .
$$

We get the same result for $x \geq y$ and obtain

$$
\begin{aligned}
& \lim _{n \rightarrow \infty} \max _{x \in[0,1]}\left|R_{\lambda, n}^{N} f(x)-R_{\lambda}^{N} f(x)\right| \\
& \leq \lim _{n \rightarrow \infty} \max _{x \in[0,1]}\left|\int_{0}^{1} \rho_{\lambda}^{N}(x, y) f(y) \mathrm{d} \mu(y)-\int_{0}^{1} \rho_{\lambda}^{N}(x, y) f(y) \mathrm{d} \mu_{n}(y)\right| \\
& +\lim _{n \rightarrow \infty} \max _{x \in[0,1]}\left|\int_{0}^{1}\left(\rho_{\lambda}^{N}(x, y)-\rho_{\lambda, n}^{N}(x, y)\right) f(y) \mathrm{d} \mu_{n}\right| \\
& =0 .
\end{aligned}
$$


Now, let $b=D$. Again using the triangle inequality, for $n \in \mathbb{N}, x, y \in[0,1], x \leq y$,

$$
\begin{aligned}
& \left|\rho_{\lambda}^{D}(x, y)-\rho_{\lambda, n}^{D}(x, y)\right| \\
& \leq \frac{1}{\sqrt{\lambda}}\left(\left|\left(\sinh _{\sqrt{\lambda}}(1)\right)^{-1}-\left(\sinh _{\sqrt{\lambda}, n}(1)\right)^{-1}\right|\left|\sinh _{\sqrt{\lambda}}(x) \sinh _{\sqrt{\lambda}}(1-y)\right|\right. \\
& +\left|\sinh _{\sqrt{\lambda}}(x)-\sinh _{\sqrt{\lambda}, n}(x)\right|\left|\left(\sinh _{\sqrt{\lambda}, n}(1)\right)^{-1} \sinh _{\sqrt{\lambda}}(1-y)\right| \\
& \left.+\left|\sinh _{\sqrt{\lambda}}(1-y)-\sinh _{\sqrt{\lambda}, n}(1-y)\right|\left|\left(\sinh _{\sqrt{\lambda}, n}^{\prime}(1)\right)^{-1} \sinh _{\sqrt{\lambda}, n}(x)\right|\right) .
\end{aligned}
$$

We have

$$
\sinh _{\sqrt{\lambda}}(1)=\sum_{n=0}^{\infty} \lambda^{n+\frac{1}{2}} q_{2 n+1}(1) \geq \sqrt{\lambda} q_{1}(1)=\sqrt{\lambda}
$$

and thus

$$
\left|\left(\sinh _{\sqrt{\lambda}}(1)\right)^{-1}-\left(\sinh _{\sqrt{\lambda, n}}(1)\right)^{-1}\right| \leq 2 \sqrt{\lambda} e^{\lambda}\left\|F-F_{n}\right\|_{\infty} .
$$

Arguing in the same way as before, we get

$$
\begin{aligned}
\lim _{n \rightarrow \infty} \max _{x \in[0,1]}\left|\rho_{\lambda}^{D}(x, y)-\rho_{\lambda, n}^{D}(x, y)\right| & \leq \lim _{n \rightarrow \infty} \frac{2}{\sqrt{\lambda}} \sqrt{\lambda} e^{\lambda}\left\|F-F_{n}\right\|_{\infty} \lambda e^{2 \lambda} \\
& +\lim _{n \rightarrow \infty} \frac{4}{\sqrt{\lambda}} \lambda^{\frac{3}{2}} e^{\lambda}\left\|F-F_{n}\right\|_{\infty} e^{\lambda} \\
& =\lim _{n \rightarrow \infty}\left(2 e^{\lambda}+4\right) \lambda e^{2 \lambda}\left\|F-F_{n}\right\|_{\infty} \\
& =0 .
\end{aligned}
$$

Further,

$$
\begin{aligned}
& \max _{x \in[0,1]}\left|\int_{0}^{1} \rho_{\lambda}^{D}(x, y) f(y) \mathrm{d} \mu(y)-\int_{0}^{1} \rho_{\lambda}^{D}(x, y) f(y) \mathrm{d} \mu_{n}(y)\right| \\
& \leq \max _{x \in[0,1]}\left|\left(\sqrt{\lambda} \sinh _{\sqrt{\lambda}}(1)\right)^{-1} \sinh _{\sqrt{\lambda}}(x)\right| \mid \int_{0}^{1} \sinh _{\sqrt{\lambda}}(1-y) f(y) \mathrm{d} \mu(y) \\
& \quad-\int_{0}^{1} \sinh _{\sqrt{\lambda}}(1-y) f(y) \mathrm{d} \mu_{n}(y) \mid \\
& \leq\left.\left|\left(\sqrt{\lambda} \sinh _{\sqrt{\lambda}}(1)\right)^{-1}\right||| \sinh _{\sqrt{\lambda}}\right|_{\infty} \mid \int_{0}^{1} \sinh _{\sqrt{\lambda}}(1-y) f(y) \mathrm{d} \mu(y) \\
& \quad-\int_{0}^{1} \sinh _{\sqrt{\lambda}}(1-y) f(y) \mathrm{d} \mu_{n}(y) \mid .
\end{aligned}
$$


Due to the weak measure convergence, this goes to zero as $n$ tends to $\infty$. Deducing the same result for $x \geq y$ and combining the above inequalities,

$$
\begin{aligned}
\lim _{n \rightarrow \infty} \max _{x \in[0,1]} \mid & R_{\lambda, n}^{D} f(x)-R_{\lambda}^{D} f(x) \mid \\
& \leq \lim _{n \rightarrow \infty} \max _{x \in[0,1]}\left|\int_{0}^{1} \rho_{\lambda}^{D}(x, y) f(y) \mathrm{d} \mu(y)-\int_{0}^{1} \rho_{\lambda}^{D}(x, y) f(y) \mathrm{d} \mu_{n}(y)\right| \\
& +\lim _{n \rightarrow \infty} \max _{x \in[0,1]}\left|\int_{0}^{1}\left(\rho_{\lambda}^{D}(x, y)-\rho_{\lambda, n}^{D}(x, y)\right) f(y) \mathrm{d} \mu_{n}\right| \\
& =0 .
\end{aligned}
$$

Remark 5.5. We made the assumption $\operatorname{supp}(\mu) \subseteq \operatorname{supp}\left(\mu_{n}\right)$ for all $n \in \mathbb{N}$ only to simplify the proofs. Note that our results can be formulated and proven in a very similar way if this condition is not satisfied. If, for example, $\operatorname{supp}(\mu) \supseteq \operatorname{supp}\left(\mu_{n}\right)$ for all $n \in \mathbb{N}$, define $\pi_{n}:(C[0,1])_{\mu}^{b} \rightarrow(C[0,1])_{\mu_{n}}^{b},\left.f \mapsto f\right|_{\operatorname{supp}\left(\mu_{n}\right)}$. This is again a bounded linear transformation between Banach spaces and we can follow the same steps as before.

\subsection{Strong semigroup convergence}

Let $\mu$ be defined as before and let $\lambda>0$. Analogously to the restricted semigroup, we define the restricted resolvent operator by

$$
\begin{gathered}
\bar{R}_{\lambda}^{N}:(C[0,1])_{\mu}^{N} \rightarrow(C[0,1])_{\mu}^{N}, \bar{R}_{\lambda}^{N} f=R_{\lambda}^{N} f, \\
\bar{R}_{\lambda}^{D}:(C[0,1])_{\mu}^{D} \rightarrow(C[0,1])_{\mu}^{D}, \bar{R}_{\lambda}^{D} f=R_{\lambda}^{D} f .
\end{gathered}
$$

Further, we define the operators $\bar{\Delta}_{\mu}^{N}$ and $\bar{\Delta}_{\mu}^{D}$ by

$$
\begin{aligned}
& \bar{\Delta}_{\mu}^{N} f:=\Delta_{\mu}^{N} f, \mathcal{D}\left(\bar{\Delta}_{\mu}^{N}\right):=\left\{f \in \mathcal{D}\left(\Delta_{\mu}^{N}\right): \Delta_{\mu}^{N} f \in(C[0,1])_{\mu}^{N}\right\}, \\
& \bar{\Delta}_{\mu}^{D} f:=\Delta_{\mu}^{D} f, \mathcal{D}\left(\bar{\Delta}_{\mu}^{D}\right):=\left\{f \in \mathcal{D}\left(\Delta_{\mu}^{D}\right): \Delta_{\mu}^{D} f \in(C[0,1])_{\mu}^{D}\right\},
\end{aligned}
$$

which are called the part of the operator $\Delta_{\mu}^{N}$ in $\left.C[0,1]\right)_{\mu}^{N}$ and the part of the operator $\Delta_{\mu}^{D}$ in $\left.C[0,1]\right)_{\mu}^{D}$, respectively. The following Lemma shows how the restricted semigroup, the restricted resolvent and the part of the operator are connected. For that, let $b \in\{N, D\}$.

Lemma 5.6. (i) The infinitesimal generator of the strongly continuous contraction semigroup $\left(\bar{T}_{t}^{b}\right)_{t \geq 0}$ is $\bar{\Delta}_{\mu}^{b}$.

(ii) $\bar{R}_{\lambda}^{b}$ is the resolvent of $\bar{\Delta}_{\mu}^{b}$.

Proof. For all $f \in L^{2}([0,1], \mu)$, we have $\|f\|_{\infty} \geq\|f\|_{\mu}$, therefore the inclusion map $i:(C[0,1])_{\mu}^{b} \rightarrow L^{2}([0,1], \mu), f \mapsto f$ is continuous. Moreover, $\left(\bar{T}_{t}^{b}\right)_{t \geq 0}$ defines a strongly continuous contraction semigroup on $(C[0,1])_{\mu}^{b}$ and $(C[0,1])_{\mu}^{b}$ is $\left(\bar{T}_{t}^{b}\right)_{t \geq 0^{-}}$ invariant (see Corollary 4.5). We thus can apply [9, II.2.3 Proposition] to verify (i). 
We turn to part (ii). Let $\lambda>0$ and let $\widetilde{R}_{\lambda}^{b}$ be the resolvent of $\bar{\Delta}_{\mu}^{b}$. By part (i) and [9, I.1.10 Theorem], this operator is well-defined and given by

$$
\widetilde{R}_{\lambda}^{b} f=\int_{0}^{\infty} e^{-\lambda s} \bar{T}_{s}^{b} f \mathrm{~d} s, f \in(C[0,1])_{\mu}^{b} .
$$

Further, by definition of $\left(\bar{T}_{t}^{b}\right)_{t \geq 0}$ and $\bar{R}_{\lambda}^{b}$,

$$
\bar{R}_{\lambda}^{b} f=R_{\lambda}^{b} f=\int_{0}^{\infty} e^{-\lambda s} T_{s}^{b} f \mathrm{~d} s=\int_{0}^{\infty} e^{-\lambda s} \bar{T}_{s}^{b} f \mathrm{~d} s, f \in(C[0,1])_{\mu}^{b} .
$$

It follows $\widetilde{R}_{\lambda}^{b}=\bar{R}_{\lambda}^{b}$ on $(C[0,1])_{\mu}^{b}$.

We are now able to establish strong semigroup convergence. To this end, let $\left(\mu_{n}\right)_{n \in \mathbb{N}}$ satisfy Assumption 1.1 and we assume $\operatorname{supp}(\mu) \subseteq \operatorname{supp}\left(\mu_{n}\right)$ for all $n \in \mathbb{N}$. For $b \in\{N, D\}$ let $\left(T_{t}^{b}\right)_{t \geq 0}$ be defined by $\mu,\left(T_{t, n}^{b}\right)_{t \geq 0}$ be defined by $\mu_{n}$ and analogously the restricted semigroups $\left(\bar{T}_{t}^{b}\right)_{t \geq 0}$ and $\left(\bar{T}_{t, n}^{b}\right)_{t \geq 0}$ be defined by $\mu$ and $\mu_{n}$, respectively.

Proof of Theorem 1.2. For $n \in \mathbb{N}$, $\pi_{n}$ is a bounded linear transformation between Banach spaces. Further, $\left(\bar{T}_{t}^{b}\right)_{t \geq 0}$ and $\left(\bar{T}_{t, n}^{b}\right)_{t \geq 0}, n \in \mathbb{N}$ are strongly continuous contraction semigroups on their respective spaces (see Corollary 4.5). Hence, due to the first Trotter-Kato approximation theorem (see [10, Theorem I.6.1, Problem I.8.13] for a suitable version), the assertion is a direct consequence of Theorem 5.4.

Remark 5.7. As a further direct consequence of the Trotter-Kato approximation theorem, we obtain the graph norm convergence. That is, for $f \in \mathcal{D}\left(\bar{\Delta}_{\mu}^{b}\right)$ there exists $\left(f_{n}\right)_{n \in \mathbb{N}}$ with $f_{n} \in \mathcal{D}\left(\bar{\Delta}_{\mu_{n}}^{b}\right)$ such that for $n \in \mathbb{N}$

$$
\lim _{n \rightarrow \infty}\left\|\pi_{n} f-f_{n}\right\|_{\infty}+\left\|\pi_{n} \bar{\Delta}_{\mu}^{b} f-\bar{\Delta}_{\mu_{n}}^{b} f_{n}\right\|_{\infty}=0 .
$$

Strong semigroup convergence can be interpreted as convergence of solutions to heat equations. The connection is given as follows (see [9, Proposition VI.6.2]).

Lemma 5.8. Let $A$ be the generator of a strongly continuous semigroup $\left(S_{t}\right)_{t \geq 0}$ on a Banach space $X$. Then, for each $f \in \mathcal{D}(A)$ the abstract heat equation

$$
\begin{aligned}
\frac{\partial u}{\partial t}(t) & =A u(t), t \geq 0 \\
u(0) & =f
\end{aligned}
$$

has a unique classical solution on $X$ given by

$$
u:[0, \infty) \rightarrow X, t \mapsto S_{t} f
$$

meaning that $u$ is continuously differentiable with respect to $X, u(t) \in \mathcal{D}(A)$ and (21) holds for all $t \geq 0$. 
Let $T>0$ and $f \in \mathcal{D}\left(\bar{\Delta}_{\mu}^{b}\right)$. Theorem 1.2 implies that the classical solution to

$$
\begin{aligned}
\frac{\partial u_{n}}{\partial t}(t) & =\bar{\Delta}_{\mu_{n}}^{b} u_{n}(t), \\
u_{n}(0) & =\pi_{n} f
\end{aligned}
$$

converges uniformly for $(t, x) \in[0, T] \times[0,1]$ to the classical solution to

$$
\begin{aligned}
\frac{\partial u}{\partial t}(t) & =\bar{\Delta}_{\mu}^{b} u(t), \\
u(0) & =f
\end{aligned}
$$

as $n \rightarrow \infty$, assuming that $\pi_{n} f \in \mathcal{D}\left(\bar{\Delta}_{\mu_{n}}^{b}\right)$. However, the assumption $f \in \mathcal{D}\left(\bar{\Delta}_{\mu}^{b}\right)$ and $\pi_{n} f \in \mathcal{D}\left(\bar{\Delta}_{\mu_{n}}^{b}\right)$ for all $n \in \mathbb{N}$ is very restrictive, as the following example illustrates.

Example 5.9. Let $\mu$ be a measure satisfying our conditions such that $\operatorname{supp}(\mu)$ is a $\lambda^{1}$-zero set and assume that $\operatorname{supp}\left(\mu_{n}\right)=[0,1]$ for all $n \in \mathbb{N}$. Further, let $f \in \mathcal{D}\left(\bar{\Delta}_{\mu}^{b}\right)$. Then, on any interval $I \subseteq[0,1] \backslash \operatorname{supp}(\mu), \pi_{n} f$ is affine. Now, if we assume that $\pi_{n} f \in \mathcal{D}\left(\bar{\Delta}_{\mu_{n}}^{b}\right)$, then $\bar{\Delta}_{\mu_{n}}^{b} f(x)=0, x \in I$ and thus $\bar{\Delta}_{\mu_{n}}^{b} f=0 \in(C[0,1])_{\mu_{n}}^{b}$. If $b=D$, we obtain $\pi_{n} f=0 \in(C[0,1])_{\mu_{n}}^{D}$ and thus $f=0 \in(C[0,1])_{\mu}^{b}$ and if $b=N$, $\left(\pi_{n} f\right)^{\prime}=0 \in C[0,1]$ and thus $f^{\prime}=0 \in(C[0,1])_{\mu}^{N}$.

This motivates the concept of a mild solution (see [10, Definition II.6.3]).

Definition 5.10. Let $X$ be a Banach space, $A: \mathcal{D}(A) \subset X \rightarrow X$ and $f \in X$. We call a map $u:[0, \infty) \rightarrow X, t \mapsto u(t)$ a mild solution to the abstract heat equation

$$
\begin{aligned}
\frac{\mathrm{d} u}{\mathrm{~d} t}(t) & =A u(t), t \geq 0, \\
u(0) & =f
\end{aligned}
$$

if $\int_{0}^{t} u(s) d s \in \mathcal{D}(A)$ and $u(t)=A \int_{0}^{t} u(s) d s+f$ for all $t \geq 0$

Using this solution concept, we can establish the desired convergence for any initial condition in the appropriate space.

Theorem 5.11. Let $f \in(C[0,1])_{\mu}^{b}$ and let $\left(\mu_{n}\right)_{n \in \mathbb{N}}$ satisfy Assumption 1.1. Further, let $\{u(t): t \geq 0\}$ be the unique mild solution to

$$
\begin{aligned}
\frac{\mathrm{d} u}{\mathrm{~d} t}(t) & =\bar{\Delta}_{\mu}^{b} u(t), t \geq 0, \\
u(0) & =f
\end{aligned}
$$

and, for $n \geq 1$, let $\left\{u_{n}(t): t \geq 0\right\}$ be the unique mild solution to

$$
\begin{aligned}
\frac{\mathrm{d} u_{n}}{\mathrm{~d} t}(t) & =\bar{\Delta}_{\mu_{n}}^{b} u_{n}(t), t \geq 0, \\
u_{n}(0) & =\pi_{n} f .
\end{aligned}
$$


Then,

$$
\lim _{n \rightarrow \infty}\left\|\pi_{n} u(t)-u_{n}(t)\right\|_{\infty}=0
$$

uniformly on bounded time intervals.

Proof. Since $\bar{\Delta}_{\mu}^{b}$ is the generator of a strongly continuous semigroup, it follows by [10, Proposition II.6.4] that $t \mapsto \bar{T}_{t}^{b} f$ is the unique mild solution to (23). The same argument show that $\bar{T}_{t, n}^{b} \pi_{n} f$ is the unique mild solution to (24) for $n \in \mathbb{N}$. Then, (25) is a direct consequence of Theorem 1.2.

\section{Applications}

Example 6.1. As a first application, we consider a non-atomic Borel probability measure $\mu$ on $[0,1]$ such that $0,1 \in \operatorname{supp}(\mu)$ and $\operatorname{supp}(\mu) \neq[0,1]$. We define for $\varepsilon \in(0,1)$ the approximating probability measure $\mu_{\varepsilon}$ by

$$
\mu_{\varepsilon}:=\frac{\mu+\varepsilon \lambda^{1}}{1+\varepsilon} .
$$

It is elementary that $\mu_{\varepsilon}$ converges weakly to $\mu$ as $\varepsilon \rightarrow 0$ and Theorem 5.11 is applicable. Let $b \in\{N, D\}$ and $f \in(C[0,1])_{\mu}^{b}$. Then, the unique solution $\left\{u_{\varepsilon}(t)\right.$ : $t \geq 0\}$ to

$$
\begin{aligned}
\frac{\mathrm{d} u_{\varepsilon}}{\mathrm{d} t}(t) & =\bar{\Delta}_{\mu_{\varepsilon}}^{b} u_{\varepsilon}(t), \\
u_{\varepsilon}(0) & =\pi_{\varepsilon} f,
\end{aligned}
$$

where $\pi_{\varepsilon}:(C[0,1])_{\mu}^{b} \rightarrow(C[0,1])_{\mu_{\varepsilon}}^{b}$ is an embedding as previously defined (see (17)), converges to the unique solution $\{u(t): t \geq 0\}$ to

$$
\begin{aligned}
\frac{\mathrm{d} u}{\mathrm{~d} t}(t) & =\bar{\Delta}_{\mu}^{b} u(t), \\
u(0) & =f
\end{aligned}
$$

with respect to the uniform norm as $\varepsilon$ tends to zero.

In the previous example, $\mu$ could be chosen to be an absolutely continuous measure, for example $\lambda_{\mid\left[0, \frac{1}{3}\right] \cup\left[\frac{2}{3}, 1\right]}^{1}$, or to be a singular measure, as a self-similar measure on the Cantor set. Furthermore, it is not required that the approximating measures have full support.

Example 6.2. Let $w_{1}, w_{2} \in(0,1)$ such that $w_{1}+w_{2}=1$ and let $\mu$ be the unique invariant Borel probabiliy measure on $[0,1]$ given by the IFS consisting of $S_{1}(x)=\frac{x}{3}$ and $S_{2}(x)=\frac{2}{3}+\frac{x}{3}, x \in[0,1]$ and weights $w_{1}$ and $w_{2}$, i.e., $\mu$ is a so-called Cantor 

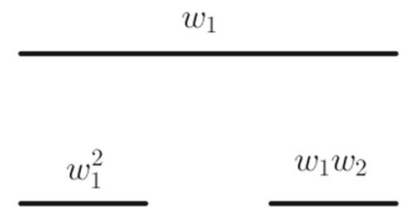

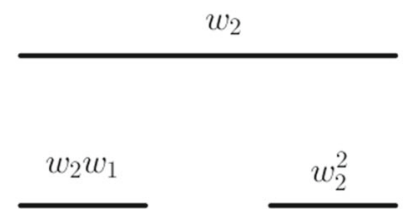

Figure 1. Approximating Cantor measures of levels $n=0,1,2$

measure. Following [19], for $n \in \mathbb{N}$ we define the approximating Cantor measures of level $n$ by

$$
\mu_{n}(B):=3^{n} \sum_{x \in\{1,2\}^{n}} \lambda_{\left.\right|_{I_{x}}}^{1} \prod_{i=1}^{n} \omega_{x_{i}}, B \in B([0,1]),
$$

where $I_{x}:=\left(S_{x_{1}} \circ \cdots \circ S_{x_{n}}\right)([0,1]), x \in\{1,2\}^{n}$. The approximating Cantor measures of levels $n=0,1,2$ are illustrated in Fig. 1 . We denote the distribution function of $\mu$ by $F$ and the distribution function of $\mu_{n}$ by $F_{n}$ for $n \in \mathbb{N}$. Then, $\left\|F-F_{n}\right\|_{\infty} \rightarrow 0$ (see [19, Proposition 4.2]) as well as $\operatorname{supp}(\mu) \subset \operatorname{supp}\left(\mu_{n}\right)$ for $n \in \mathbb{N}$ and Theorem 5.11 can be applied. Hence, for $f \in(C[0,1])_{\mu}^{b}$, the unique solution $\left\{u_{n}(t): t \geq 0\right\}$ to

$$
\begin{aligned}
\frac{\mathrm{d} u_{n}}{\mathrm{~d} t}(t) & =\bar{\Delta}_{\mu_{n}}^{b} u_{n}(t), \\
u_{n}(0) & =\pi_{n} f
\end{aligned}
$$

converges to the unique solution $\{u(t): t \geq 0\}$ to

$$
\begin{aligned}
\frac{\mathrm{d} u}{\mathrm{~d} t}(t) & =\bar{\Delta}_{\mu}^{b} u(t), \\
u(0) & =f
\end{aligned}
$$

with respect to the uniform norm as $n$ tends to infinity.

Finally, we connect both applications.

Example 6.3. Let $\varepsilon>0, n \in \mathbb{N}$ and let $\mu, \mu_{n},\{u(t): t \geq 0\}$ and $\left\{u_{n}(t): t \geq 0\right\}$ be defined as in Example 6.2. We define $\mu_{n, \varepsilon}$ by

$$
\mu_{n, \varepsilon}:=\frac{\mu_{n}+\varepsilon \lambda^{1}}{1+\varepsilon}
$$

i.e. analogously to Example 6.1, and $\left\{u_{n, \varepsilon}(t): t \geq 0\right\}$ to be the solution to

$$
\begin{aligned}
\frac{\mathrm{d} u_{n, \varepsilon}}{\mathrm{d} t}(t) & =\bar{\Delta}_{\mu_{n, \varepsilon}}^{b} u_{n, \varepsilon}(t), \\
u_{n, \varepsilon}(0) & =\pi_{n, \varepsilon} f,
\end{aligned}
$$


where $\pi_{n, \varepsilon}$ is an embedding as previously defined. Further, let $t \in[0, \infty)$ and $\delta>0$. By Example 6.2, there exists $n_{0} \in \mathbb{N}$ such that for all $n \geq n_{0}$ we have

$$
\left\|u(t)-u_{n}(t)\right\|_{\infty}<\frac{\delta}{2} .
$$

By Example 6.1, for each $n \geq n_{0}$ there exists $\varepsilon_{n}>0$ such that for all $\varepsilon<\varepsilon_{n}$ we have

$$
\left\|u_{n}(t)-u_{n, \varepsilon}(t)\right\|_{\infty}<\frac{\delta}{2} .
$$

Hence, for all $n \geq n_{0}, \varepsilon<\varepsilon_{n}$ we have

$$
\left\|u(t)-u_{n, \varepsilon}(t)\right\|_{\infty}<\delta
$$

Thus, the heat in a rod with mass distribution given by a Cantor measure diffuses approximately like the heat on a rod possessing a strictly positive mass density which is small off the Cantor set.

\section{Directions for further research}

Remark 7.1. Consider the heat equation (2) with initial value given by the Delta distribution $\delta_{y}: g \mapsto g(y)$ for $y \in \operatorname{supp}(\mu)$. Then, the heat kernel

$$
p_{t}(x, y)=\sum_{k \geq 1} e^{-\lambda_{k}^{b} t} \varphi_{k}^{b}(x) \varphi_{k}^{b}(y),(t, x) \in[0, \infty) \times(0,1]
$$

solves the equation in the distributional sense, where $\left\{\lambda_{k}^{b}, k \geq 1\right\}$ are the ascending ordered eigenvalues and $\left\{\varphi_{k}^{b}, k \geq 1\right\}$ the $L_{2}([0,1], \mu)$-normed eigenfunctions of $\Delta_{\mu}^{b}$ on $L_{2}([0,1], \mu)$. The heat kernel is of particular importance in the context of the associated Markov process (see the remark below) and stochastic partial differential equations (see [7,8]). It is an open question whether weak measure convergence implies pointwise convergence of the corresponding heat kernels for each $(t, x, y) \in(0, \infty) \times$ $[0,1]^{2}$.

Remark 7.2. The operator $\Delta_{\mu}^{b}$ on $L_{2}([0,1], \mu)$ is the infinitesimal generator of a Markov process, called a quasi-diffusion (see, e.g., [27,30-32]). Convergence of semigroups raises the question of whether the associated Markov processes also converge weakly. If $\mu_{n} \rightarrow \mu$, our results imply that for each $f \in(C[0,1])_{\mu}^{b}, t \in[0, \infty)$ and each starting point $x \in[0,1]$

$$
\mathbb{E}\left[f\left(X_{n}^{b}(t)\right)\right]=T_{t, n}^{b} f(x) \rightarrow T_{t}^{b} f(x)=\mathbb{E}\left[f\left(X^{b}(t)\right)\right], n \rightarrow \infty,
$$

where $X^{b}$ is associated to $\Delta_{\mu}^{b}$ and $X_{n}^{b}$ is associated to $\Delta_{\mu_{n}}^{b}$. We denote by $\mathbb{E}$ the expectation with respect to the law of the appropriate process. This could be extended to a proof of convergence of all finite-dimensional distributions, and tightness would then also be required in order to establish that $X_{n}^{b} \rightarrow X^{b}$ weakly in the Skorokhod space of càdlàg functions. 
Remark 7.3. Let $\mu$ be of full support. Consider the analogue of the wave equation

$$
\frac{\mathrm{d}^{2} u}{\mathrm{~d} t^{2}}(t)=\Delta_{\mu}^{b} u(t), t \in[0, \infty)
$$

on $L^{2}([0,1], \mu)$. This hyperbolic equation describes the motion of a vibrating string with mass distribution $\mu$ such that, if it is deflected, a tension force drives it back towards its state of equilibrium. If $\mu$ were not of full support, the string would have massless parts. It is not clear how to interpret massless parts of a string. We suppose that the motion of such a string behaves approximately like the motion of a string with very little mass on these gaps, analogous to our results about the diffusion of heat.

Assume that $u(0) \in \mathcal{D}\left(\Delta_{\mu}^{b}\right)$ and, for reasons of simplicity, that the initial velocity vanishes. Then, there exists a unique solution on $L_{2}([0,1], \mu)$ given by $u(t)=$ $C(t) u(0), t \geq 0$, where $\{C(t): t \geq 0\}$ denotes the strongly continuous cosine family of $\Delta_{\mu}^{b}$ (see, e.g., [40]). We have already shown that $\mu_{n} \rightarrow \mu$ implies strong resolvent convergence of the corresponding operators restricted to continuous functions. It is well-known that this implies convergence of the corresponding cosine families $\left\{C_{n}(t): t \geq 0\right\}$, which implies convergence of the solutions to the corresponding wave equation, provided that there exists $M>0$ and $w \geq 0$ such that for all $n \geq 1, t \geq 0$ $\left\|C_{n}(t)\right\| \leq M e^{w|t|}$ (see [25]). Proving that the restriction of $C(t)$ to $(C[0,1])_{\mu}^{b}$ is the cosine family of $\bar{\Delta}_{\mu}^{b}$ (and analogously for $\mu_{n}$ ) and verifying the above estimate would be a way to establish the desired convergence of solutions to the wave equation.

Remark 7.4. The Krein-Feller operator $\Delta_{\mu}^{b}$ can also be defined with respect to a measure $\mu$ with atoms, see, e.g., [29] for a model in this direction. If $\mu$ is atomless, the weak convergence implies uniform convergence of the corresponding distribution functions, which we have used to prove convergence of the corresponding generalized monomials (see Remark 5.2). For a measure with atoms, it would be required to find such convergence results without this uniform convergence property. Further, a representation of the resolvent density needs to be developed (see Lemma 3.1 for atomless measures).

\section{Acknowledgements}

Open Access funding provided by Projekt DEAL. The authors thank the referee for very helpful suggestions for improvement.

Open Access. This article is licensed under a Creative Commons Attribution 4.0 International License, which permits use, sharing, adaptation, distribution and reproduction in any medium or format, as long as you give appropriate credit to the original author(s) and the source, provide a link to the Creative Commons licence, and indicate if changes were made. The images or other third party material in this article are included in the article's Creative Commons licence, unless indicated otherwise in a credit line to the material. If material is not included in the article's Creative Commons licence and your intended use is not permitted by statutory regulation or exceeds the permitted use, you will need to obtain permission 
directly from the copyright holder. To view a copy of this licence, visit http://creativecommons.org/licenses/ by $/ 4.0 /$.

Publisher's Note Springer Nature remains neutral with regard to jurisdictional claims in published maps and institutional affiliations.

\section{REFERENCES}

[1] P. Arzt, Measure Theoretic Trigonometric Functions, Journal of Fractal Geometry, 2(2):115-169, 2015.

[2] E. J. Bird, S.-M. Ngai, A. Teplyaev, Fractal Laplacians on the Unit Interval, Ann. Sci. Math. Québec, 27:135-168, 2003.

[3] J. Chen, S.-M. Ngai, Eigenvalues and eigenfunctions of one-dimensional fractal Laplacians defined by iterated function systems with overlaps, Anal. Appl., 364:222-241, 2010.

[4] Y. S. Chow, H. Teicher, Probability Theory, 3rd ed., Springer, New York, 1997.

[5] D. Conus, M. Joseph, D. Khoshnevisan, S. Shiu, Intermittency and Chaos for a Non-linear Stochastic Wave Equation in Dimension 1, Malliavin Calc. Stoch. Ana., 34:251-279, Springer, Boston, 2013.

[6] D. A. Croydon, Scaling limits of stochastic processes associated with resistance forms, Ann. Inst. H. Poincarè Probab. Statist., 54(4):1939-1968, 2018.

[7] T. Ehnes, Stochastic Heat Equations defined by Fractal Laplacians on Cantor-like Sets, arXiv:1902.02175, 2019.

[8] T. Ehnes, Stochastic Wave Equations defined by Fractal Laplacians on Cantor-like Sets, arXiv:1910.08378, 2019.

[9] K.-J. Engel, R. Nagel, One-Parameter Semigroups for Linear Evolution Equations, 1st ed., Springer, New York, 2000.

[10] S. N. Ethier, T. G. Kurtz, Markov Processes: Characterization and Convergence, 1st ed., John Wiley, New York, 1986.

[11] W. Feller, Generalized second order differential operators and their lateral conditions, Illinois journal of mathematics, 1:459-50, 1957.

[12] U. Freiberg: A Survey on Measure Geometric Laplacians on Cantor Like Sets, Arabian Journal for Science and Engineering, 28(1C):189-198, 2003.

[13] U. Freiberg, Analytical properties of measure geometric Krein-Feller-operators on the real line, Mathematische Nachrichten, 260:34-47, 2003.

[14] U. Freiberg, Dirichlet forms on fractal subsets of the real line, Real Anal. Exchange, 30(2):589-603, 2004/05.

[15] U. Freiberg, Prüfer angle methods in spectral analysis of Krein-Feller-operators, RIMS Kôkŷ̂roku Bessatsu B6, 74-81, 2008.

[16] U. Freiberg, Refinement of the spectral asymptotics of generalized Krein Feller operators, Forum Math., 23:427-445, 2011.

[17] U. Freiberg, Spectral asymptotics of generalized measure geometric Laplacians on Cantor like sets, Forum Math., 17:87-104, 2005.

[18] U. Freiberg, J. Löbus, Zeros of eigenfunctions of a class of generalized second order differential operators on the Cantor set, Mathematische Nachrichten, 265:3-14, 2004.

[19] U. Freiberg, L. Minorics: Eigenvalue Approximation for Krein-Feller-Operators w.r.t. Invariant Singular Measures, 1903.00215, 2019.

[20] U. Freiberg, N. V. Rastegaev, On spectral asymptotics of the Sturm-Liouville problem with selfconformal singular weight, Zap. Nauchn. Sem. POMI, 477:129-135, 2018.

[21] U. Freiberg, C. Seifert, Dirichlet forms for singular diffusion in higher dimensions, J. Evol. Equ., 15:869-878, 2015.

[22] U. Freiberg, M. Zähle, Harmonic calculus on fractals-a measure geometric approach I, Potential Anal. 16:265-277, 2002.

[23] T. Fujita:A fractional dimension, self similarity and a generalized diffusion operator, Taniguchi Symp. PMMP Katata, 83-90, Kinokuniya, 1987. 
[24] M. Fukushima, Y. Oshima, M. Takeda Dirichlet forms and symmetric Markov processes, De Gruyter Stud. Math. 19, de Gruyter, Berlin-New York, 2011.

[25] J. A. Goldstein: On the convergence and approximation of cosine functions, Aeq. Math., 10(23):201-205, 1987.

[26] R. Habermann, Elementary Applied Partial Differential Equations with Fourier Series and Boundary Value Problems, 2nd ed., Prentice-Hall, New Jersey, 1987.

[27] K. Itô, H. P. Jr. McKean, Diffusion Processes and their Sample Paths, Springer-Verlag, BerlinHeidelberg-New York, 1965.

[28] J. Kigami, Analysis on Fractals (Cambridge Tracts in Mathematics), Cambridge University Press, Cambridge, 2001.

[29] U. Kant, T. Klauß, J. Voigt, M. Weber, Dirichlet forms for singular one-dimensional operators and on graphs, J. Evol. Equ., 9:637-659, 2009.

[30] U. Küchler, On sojourn times, excursions and spectral measures connencted with quasidiffusions, J. Math. Kyoto Univ., 26(3):403-421, 1986.

[31] U. Küchler, Some Asymptotic Properties of the Transition Densities of One-Dimensional Quasidiffusions, Publ. RIMS, Kyoto Univ., 16:245-268, 1980.

[32] J.-U. Löbus, Constructions and generators of one-dimensional quasidiffusions with applications to selfaffine diffusions and Brownian motion on the Cantor set, Stoch. Stoch. Rep., 42(2):93-114, 1993.

[33] L. Minorics, Spectral Asymptotics for Krein-Feller-operators with respect to random recursive Cantor measures, 1709.07291, 2017.

[34] L. Minorics, Spectral Asymptotics for Krein-Feller-Operators with respect to V-Variable Cantor Measures, 1808.06950, 2018.

[35] K. Naimark, M. Solomyak, The eigenvalue behaviour for the boundary value problems related to self-similar measures on $R^{d}$, Math. Res. Lett., 2:279-298, 1995.

[36] S.-M. Ngai, Spectral asymptotics of Laplacians associated with one-dimensional iterated function systems with overlaps, Canad. J. Math., 63:648-688, 2011.

[37] S.-M. Ngai, W. Tang, Y. Xie, Spectral asymptotics of one-dimensional fractal Laplacians in the absence of second-order identities, Discrete Contin. Dyn. Syst., 38:1849-1887, 2018.

[38] A. Pazy, Semigroups of Linear Operators and Applications to Partial Differential Equations, 1st ed., Springer, New York, 1983.

[39] M. Solomyak and E. Verbitsky, On a spectral problem related to self-similar measures, Bull. London Math. Soc., 27(3):242-248, 1995.

[40] G. F. Webb, A representation formula for strongly continuous cosine families, Aeq. Math., 21:251256,1980

\author{
Tim Ehnes \\ Institute for Stochastics and \\ Applications \\ University of Stuttgart \\ Pfaffenwaldring 57 \\ 70569 Stuttgart \\ Germany \\ E-mail:tim.ehnes@mathematik.uni-stuttgart.de \\ Ben Hambly \\ Mathematical Institute \\ University of Oxford \\ Woodstock Road \\ Oxford OX2 $6 G G$ \\ $U K$ \\ E-mail:hambly@maths.ox.ac.uk
}

